\title{
An Efficient PET-MRI Medical Image Fusion based on IHS-NSCT-PCA Integrated Method
}

\author{
Padmavathi K, Maya V Karki
}

\begin{abstract}
Merging of multiple imaging modalities leads to a single image that acquire high information content. These find useful applications in disease diagnosis and treatment planning. IHS-PCA method is a spatial domain approach for fusion that offersfinestvisibility but demands vast memory and it lacks steering information. We propose an integrated approach that incorporates NSCT combined with PCA utilizing IHS space and histogram matching. The fusion algorithm is applied on MRI with PET image and improved functional property was obtained. The IHS transform is a sharpening technique that converts multispectral image from RGB channels to Intensity Hue and Saturation independent values. Histogram matching is performed with intensity values of the two input images. Pathological details in images can be emphasized in multi-scale and multi-directions by using PCA withNSCT. Fusion rule applied is weighted averaging andprincipal components are used for dimensionality reduction. Inverse NSCT and Inverse IHS are performed so as to obtain the fused image in new RGB space. Visual and subjective investigation is compared with existing methods which demonstrate that our proposed technique gives high structural data content with high spatial and spectral resolution compared withearlier methods.
\end{abstract}

Keywords: NSCT,fusion, Histogram, IHS, PCA.

\section{INTRODUCTION}

Generating a single image by combining two or more images that integrates complementary, multitemporal or multiview information from the sources is known as image fusion. Single image acquired merges best features from each of the source images. An integration of PET and MRI can be exceptionally helpful in disease diagnosis and valid treatment scheduling [1]. The source images considered for integration are at various resolutions and intensity values. Merging various modalities of medical images help physicians to identify lesions and other features related to diseases that may not be visible in a single modality. Each of the imaging modalities has their own constraints. The individual modality does not provide all the relevant information regarding specific organs. MRI is an imaging technique that represents

\section{Revised Manuscript Received on December 30, 2019.}

* Correspondence Author

Padmavathi K.*, Department of EC, NMAM Institute of Technology, Nitte, Karnataka, Udupi, India. Email: padmavathik@nitte.edu.in

Maya V Karki, Department. of EC, Ramaiah Institute of Technology, Bengaluru, Karnataka, India. Email: mayavkarki@msrit.edu

(C) The Authors. Published by Blue Eyes Intelligence Engineering and Sciences Publication (BEIESP). This is an open access article under the CC BY-NC-ND license (http://creativecommons.org/licenses/by-nc-nd/4.0/) the internal body structures. It gives valuable information regarding smooth tissues such as brain tumors, heart, lungs and abdomen. CT provides details of bony structures with anatomical facts and high resolution. Modalities such as PET/SPECT provide functional information related to metabolism of specific tissues. PET images have color information and are with low spatial resolution. Hence more useful and valuable information is obtained by uniting anatomical and functional images that is necessary and vital[2].

In this work, MRI images considered are in gray scale while pseudo color is used to represent PET images. Fusion using IHS technique comprised of a PET image with low resolution intensity components in IHS space changed with gray level MRI image that has spatial resolution[3]. But the resultant fused image produced spectral distortion. Mutual information was high with IHS-PCA technique compared to other techniques such as DWT. Hence histogram matching was performed where high resolution data is modified such that its histogram matches that of another dataset[4]. Classification of fusion techniques are based on three levels such as pixel level, feature level and decision level[1]. Pixel level fusion has algorithms that work either in spatial domain, such as averaging, IHS method, PCA, Brovey method or in transform domain. Spatial domain approaches gives rise to spectral distortion in the combined image. To overcome this, spectral domain approaches were used toobtain fused images with better quality. DWT preserves spectral information, while the spatial characteristics are not expressed in a fine way. Shift variance at the input causes a large change at the output. Aliasing effect is present due to down sampling and non-ideal filtering all over the analysis. It lacks directional selectivity in higher dimensions. Best method is to provide shift invariance and cancel decimation process. This is achieved by using Non-Subsampled Contourlet Transform. It permits to observe dissimilar and flexible number of directions at each scale[5]. Disparate wavelets, NSCT can successfullydetain the geometrical structures such as contour edges in normal images. It is shift invariant, multi-scale, multidirectional expansion which has a firm and effective implementation. From the decomposed images, principal components are extracted and the merged imageis obtained by applying weighted averaging fusion rule. Further sections of the paper is structured as indicated. Histogram matching, IHS transform, NSCT and PCA fusion approach aredepicted in section 2 followed by suggested methodology for fusion in section 3. Section 4 describes assessment parameters. Section 5 depicts the experimental result and discussions. 


\section{FUSION MODELS}

\section{A. Histogram Matching}

Histogram matching is an effective, unsupervised and a simple technique. In this method, histogram of reference image is equalized with histogram of the second image.

Histogram matching is a problem of finding a transformation for a separate image so that its histogram accurately matches the specified histogram[6]. Several methods are suggested in the literature such as exact histogram matching and multiple histogram matching.

\section{B. IHS Transform}

Various mathematical depictions are available that can convert RGB values to parameters of human color observation. IHS transformation is an image sharpening procedure and the translation system is a linear transform. It converts a multispectral image with RGB channels to IHS components[7]. Intensity (I) shows the amount of illumination present in the spectrum. Hue $(\mathrm{H})$ labels the visual sensation of different parts of the color spectrum. Saturation (S) refers to the purity of the spectrum[4].

Since PET image is characterized in RGB space, we can segregate intensity (I), color information hue (H) and saturation (S) by using IHS transform. Here (I) component of PET may be represented as image without color information. Since (I) component of PET is similar to MRI gray image, we can match histogram of MRI image with histogram of (I) component of PET. PET has an intensity element with low resolution in IHS space. It is substituted by a high spatial resolution gray level MRI image[8],[4]. The RGB-IHS conversion model is a linear transformation.

IHS transform is a commonly used triangular spectral model[9]. The forward transform from color channels Red, Green and Blue to Intensity, Hue and Saturation are

$$
\begin{aligned}
& 0<R<1,0<G<1,0<B<1 \text {, } \\
& I=R+G+B \\
& I=\left\{\begin{array}{l}
\frac{(G-B)}{(I-3 B)} \text { if } B=\min (R, G, B) \\
\frac{(B-R)}{(I-3 R)}+1 \text { if } R=\min (R, G, B) \\
\frac{(R-G)}{(I-3 G)}+2 \text { if } G=\min (R, G, B)
\end{array}\right. \\
& S=\left\{\begin{array}{l}
\frac{(I-3 B)}{I} \text { if } 0 \leq H \leq 1 \\
\frac{(I-3 R)}{I} \text { if } 1 \leq H \leq 2 \\
\frac{(I-3 G)}{I} \text { if } 2 \leq H \leq 3
\end{array}\right. \\
& R=\left\{\begin{array}{cl}
\frac{I(1+2 S-3 S H)}{3} & \text { if } 0 \leq H \leq 1 \\
\frac{I(1-S)}{3} & \text { if } 1 \leq H \leq 2 \\
\frac{I(1-S+3 S(H-2))}{3} & \text { if } 2 \leq H \leq 3
\end{array}\right. \\
& G=\left\{\begin{array}{c}
\frac{I(1-S+3 S H)}{3} \text { if } 0 \leq H \leq 1 \\
\frac{I(1+2 S+3 S(H-1))}{3} \text { if } 1 \leq H \leq 2 \\
\frac{I(1-S)}{3} \text { if } 2 \leq H \leq 3
\end{array}\right.
\end{aligned}
$$

$$
B=\left\{\begin{array}{c}
\frac{I(1-S)}{3} \text { if } 0 \leq H \leq 1 \\
\frac{I(1-S+3 S(H-1))}{3} \text { if } 1 \leq H \leq 2 \\
\frac{I(1+2 S-3 S(H-2))}{3} \text { if } 2 \leq H \leq 3
\end{array}\right.
$$

This RGB to IHS conversion process leads to an enhanced spectral image after fusion.

\section{Non-Subsampled Contourlet Transform (NSCT)}

Intensity component of PET image and matched new MRI image are decomposed using NSCT. The NSCT is an extended version of CT[10] which is fully multiscale, multidirectional and also shift invariant. It provides better representation of contours and is also highly capable of image noise deletion and enhancement[11]. The subband decomposition of the image is similar to that of CT but in the absence of down samplers and upsamplers. NSCT is achieved with Non-Subsampled Pyramid Filter Bank (NSPFB)[12],[13].

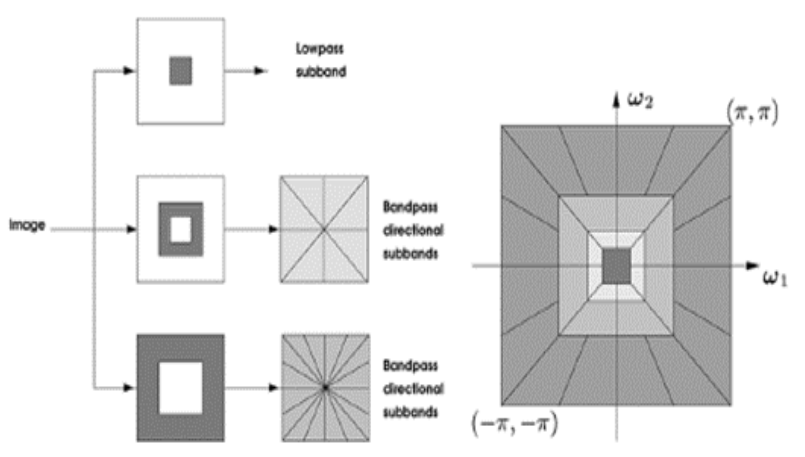

Figure 1: NonsubsampledContourlet Transform (a) NSFB used to implement NSCT (b) Frequency partitioning structure

Pyramid filter bank is used to attain Multiscale property of NSCT that uses two-channel non-subsampled filter bank. In each of the decomposition levels, the singularities in the image are captured by using one low as well as one high frequency components. The decomposition results in $M+1$ sub images with ' $M$ ' high frequency images with one low frequency image, with ' $M$ ' indicating the number of decomposition levels[14],[15].

The Non-Subsampled Directional Filter Bank consists of two channel Non-Subsampled Filter Bank. The shift invariant and multidirectional property provides directional details in the absence of downsamplers and upsamplers. The high frequency images are decomposed by DFB from NSPFB at

Each scale that creates directional sub-images with indistinguishable size as source image. Hence NSDFB presents NSCTthat has multidirectional property, better direction selection and also offers better frequency selectivity[16],[17],[18].

\section{PCA Fusion Approach}

Principal Component Analysis is a linear transformation scheme employed to highlight the data to a novel coordinate system. 
Consequently, projection on the first coordinate arranges for the greatest variance and is known as the first principal component. The next maximum variance is recognized as second principal component and so on. It permits a decrease in the number of channels by reducing the inter channel dependencies[2][4].

The two principal components from the matched new MRI image and PET intensity image can be selected based on spatial frequency (SF)[4][9].The equation for an $N \times P$ pixels image $i(x, y)$ can be defined as,

$$
S F=\sqrt{(R F)^{2}+(C F)^{2}}
$$

$R F=\sqrt{\frac{1}{N \times P} \sum_{j=1}^{P} \sum_{k=2}^{N}[i(j, k)-i(j, k-1)]^{2}}$

and CF is Column Frequency[19] given by

$C F=\sqrt{\frac{1}{N \times P} \sum_{k=1}^{N} \sum_{j=2}^{P}[i(j, k)-i(j-1,)]^{2}}$

respectively. The normalized weights ' $\alpha$ ' and ' $\beta$ ' are computed as

$\alpha=\frac{S F_{1}}{S F_{1}+S F_{2}}$

where RF is Row Frequency[19] given by

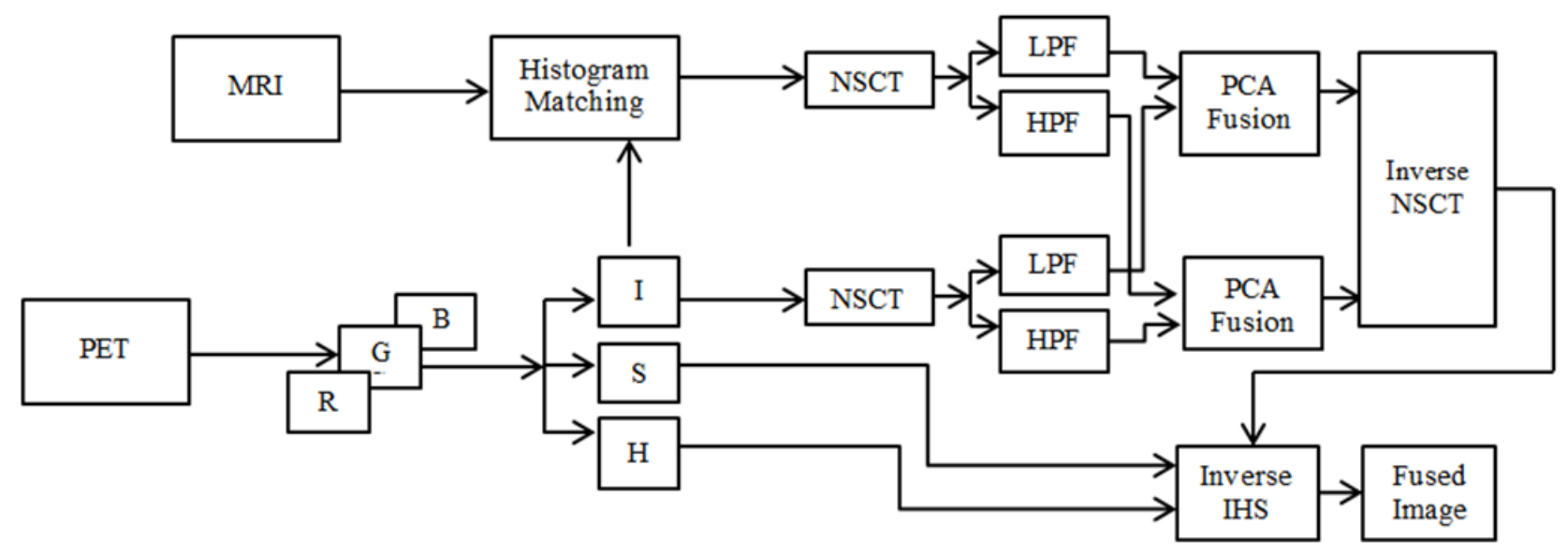

Figure 2: The proposed IHS-NSCT-PCA based integrated fusion approach

and $\beta=\frac{S F_{2}}{S F_{1}+S F_{2}}$

Fused image coefficients are obtained using weighted coefficients ' $\alpha$ ' and ' $\beta$ ' based on $\mathrm{SF}$ is given by

$I=\alpha I_{1}+\beta I_{2}$

where $I_{1}$ and $I_{2}$ represents the principal components after PCA transform.

\section{PROPOSED METHODOLOGY}

The proposed method shown in Figure 2, considers a high resolution MRI and a low resolution PET images of Alzheimer's disease and brain tumor for fusion. MRI is an intensity image while PET is in RGB format.

\section{Algorithm:}

Step 1: Transform PET image into IHS triangular model components from RGB.

Step 2: Histogram matching is performed in this step where the intensity distribution of MRI image is matched with the space.

Step 3: Decomposition of matched new MRI image and intensity component of PET image are performed by applying Non Subsampled Contourlet Transform(NSCT) to obtain low and high frequency subbands. intensity component of PET image from IHS

Step4: PCA fusion rule is applied distinctly on low frequency and high frequency sub-band coefficients of MRI and PET images.

Step 5: Inverse NSCT is applied that generates a new fused intensity image.

Step 6: Lastly, Inverse IHS is performed on newly obtained fused intensity image and old hue and saturationcomponents to achieve final fused image back in RGB space.

\section{PERFORMANCE PARAMETERS}

Performance evaluation of the proposed model can be assessed by no reference and full reference methods as below[20][21]

1) Entropy (E): Measure of data content present in combined image is estimated utilizing Entropy. Higher the entropy esteem, wealthier is the data content in the intertwined image. It is given by

$$
E=\sum_{i=0}^{L} h_{I_{f}}(i) \log _{2} h_{I_{f}}(i) \text { (13) }
$$

' $E^{\prime}$ is entropy of fused image and histogram calculation of fused image is indicated by ' $h_{I_{f}}{ }^{\prime}$.

2) Standard Deviation (SD): Standard deviation is utilized to quantify contrast of an image. Highstandard deviation of a fused image specifies that the contrast of fused image obtained is very high. 
$\sigma=\sqrt{\sum_{i=0}^{L}(i-\bar{i})^{2} h_{I_{f}}(i)}$

where, $\sigma^{\prime}$ is the standard deviation of the merged image, ' $h_{I_{f}}{ }^{\prime}$ is histogram computation of fused image, ' $i$ ' is the index of summation and ' $\bar{i}$ ' is mean of the histogram.

3) Average Gradient (AG): The edge information in an image is highlighted by this parameter. After the fusion process, it checks if edge information is reserved, lost or improved when associated to source images. It reflects the clarity of fused image. Greater the value of Average Gradient more is the clarity.

$$
A G=\frac{\sum_{i=0}^{M-1} \sum_{j=0}^{N-1} g(i, j)}{M N}
$$

where, $g(i, j)$ is the magnitude gradient at location $(i, j)$.

4) Discrepancy (D): This shows the ability of fused images to retain the spectral content present in input images. A lower value of discrepancyindicates higher spectral resolution.

$$
\begin{gathered}
D=\frac{1}{M N} \sum_{i=0}^{M-1} \sum_{j=0}^{N-1}\left|f_{k}(i, j)-f_{2 k}(i, j)\right| \\
k \rightarrow R, G \text { and } B
\end{gathered}
$$

$f_{k}(i, j), f_{2 k}(i, j)$ are pixel values of fused and original images at $(i, j)$.

5) Overall performance (O.P): This parameter is computed as difference between (AG) and Discrepancy (D). A higher overall fusion quality is depicted by small amount of overall performance. It is given by

$$
\text { O.P }=\frac{\sum_{k}|D-A G|}{3}, k \rightarrow R, G, B
$$

6) Average Difference (AD): This parameter helps in providing the information as how different is the fused image from the reference image. It is measured as the average value of difference between reference and fused image. It is specified by

$$
A D=\frac{1}{M N} \sum_{i=0}^{M-1} \sum_{j=0}^{N-1}\left[I_{r}(i, j)-I_{f}(i, j)\right]
$$

7) Structural Content (SC): It estimates the similarity measure of the structure of two images. The total weight of an original image is compared with fused image to measure the distortion contained in it. For a high quality fused image, structural content must be low and within 1 . It is given by

$S C=\frac{\sum_{i=1}^{M} \sum_{j=1}^{N} I_{r}(i, j)^{2}}{\sum_{i=1}^{M} \sum_{j=1}^{N} I_{f}(i, j)^{2}}$

where, $I_{r}(i, j)$ is the reference image with $I_{f}(i, j)$ as fused image.

8) Normalized Cross Correlation (NCC): It is used to find measure of similarity between reference image and fused image. For a good quality fused image NCC is high and lies within 1 . It is given by

$$
\sum_{i=0}^{M-1} \sum_{j=0}^{N-1} \frac{\left(I_{r_{i j}}^{* I} f_{i j}\right)}{I_{r_{i j}}{ }^{2}}
$$

\section{RESULTS AND DISCUSSIONS}

The dataset consists of 2 groups: Alzheimer's disease and brain tumor. Alzheimer's disease comprises of 23 color PET images and 23 MRI images high resolution. Brain tumor dataset comprises of 17 color PET images with 17 high resolution MRI images. The datasets are acquired from [24] http://www.med.harvard.edu/aanlib/. All images considered are of size $256 * 256$. The Brovey transform, Wavelet transform, MSVD and the proposed hybrid method were applied to merge the image datasets and the results were compared. Fused results can be evaluated visually. Other than visual evaluation, quantitative analysis is also carried out with the selected fusion algorithms by using the

parameters Entropy (EN), Standard Deviation (SD), Average Gradient (AG), Discrepancy (D), Overall Performance (OP), Average Difference (AD), Structural content (SC) and Normalized Cross Correlation (NCC).

Brovey method preserves spatial information but suffers from spectral distortion where the spectral range of input images is different. Wavelet transform decomposes the image into limited number of directions and is not shift invariant. MSVD technique performs better compared to wavelet transform[22]. While NonsubsampledContourlet Transform (NSCT) provides multiscale, multidirectional decomposition, we have proposed a hybrid technique of integrating NSCT with IHS and PCA to merge MRI and PET images. Original datasets and fused images are depicted in Figure 3 and Figure 4.

Table I and Table II show the performance analysis of fused images corresponding to Alzheimer's disease and brain tumor. In both cases, Entropy (EN) and Standard Deviation (SD) are high indicating high datacontent and high contrast in fused image of proposed method compared with other three methods. The amount of Discrepancy (D) Overall Performance (OP) and Structural Content (SC) are low for the suggested method which shows that the integrated method provides better quality fused images by preserving spectral features. Average Gradient (AG), Average Difference (AD) and Normalized Cross Correlation (NCC) is high for our method indicating the presence of edge contours and spatial features compared to other methods MATLAB R2019a software tool is used to perform simulation.Spatial domain techniques like averaging and IHS-PCA retain spatial resolution with low contrast and low data content. We have proposed a novel hybrid technique of integrating NSCT with IHS-PCA model for image fusion with histogram matching. This process preserves very high directional information, spatial information and color details of PET images. Edges and contours are also highly preserved in our method when compared with other existing methods.

The principal components selected minimize redundancy and also reduces storage space. New fused images obtained using our method has color information of PET image with spatial details of MRI image at once which gives the best results. 
(A)

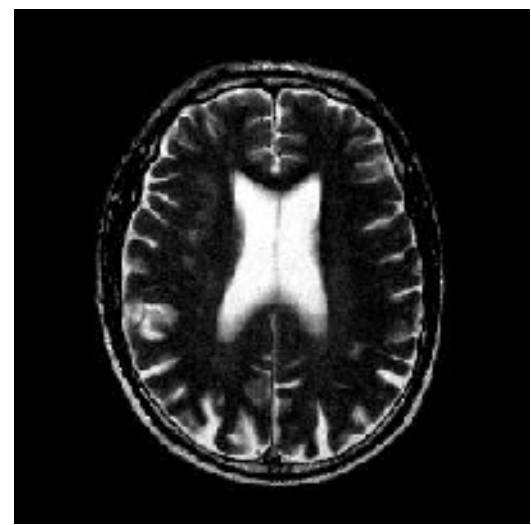

(C)

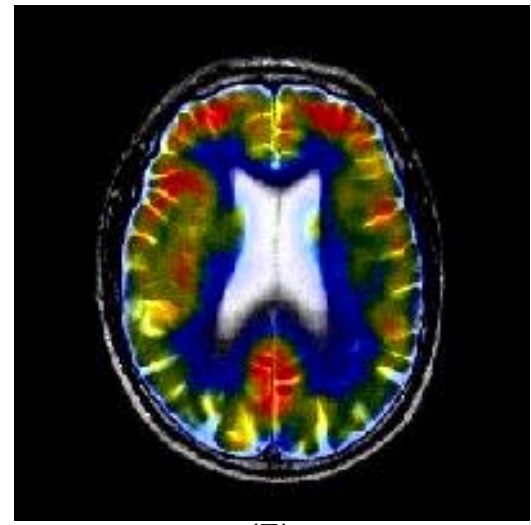

$(\mathrm{E})$

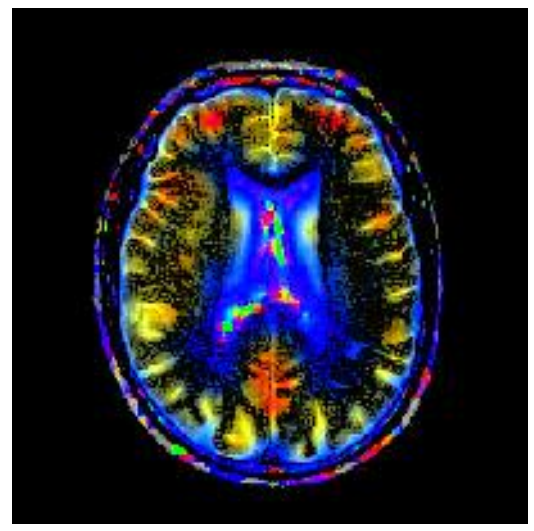

(B)

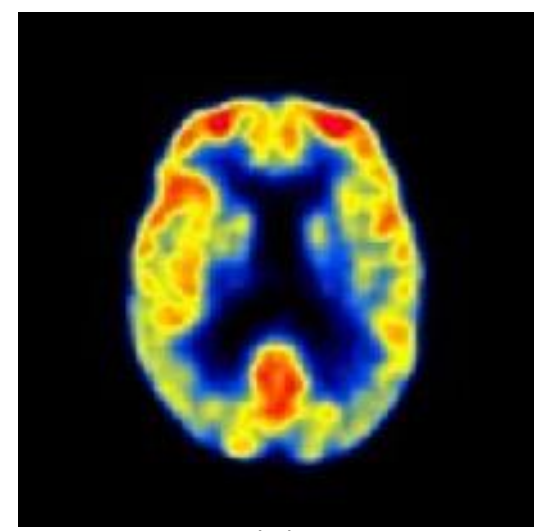

(D)

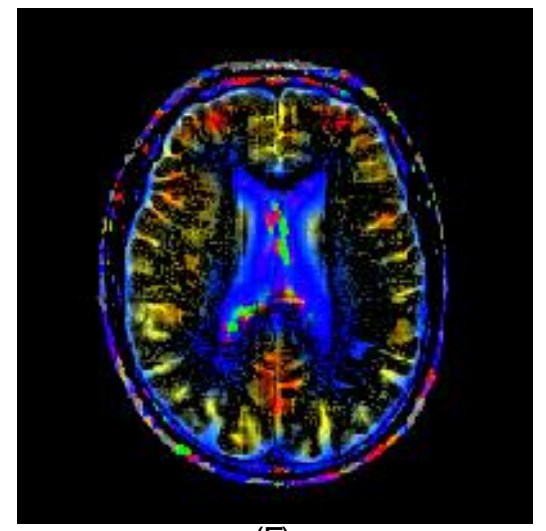

$(\mathrm{F})$

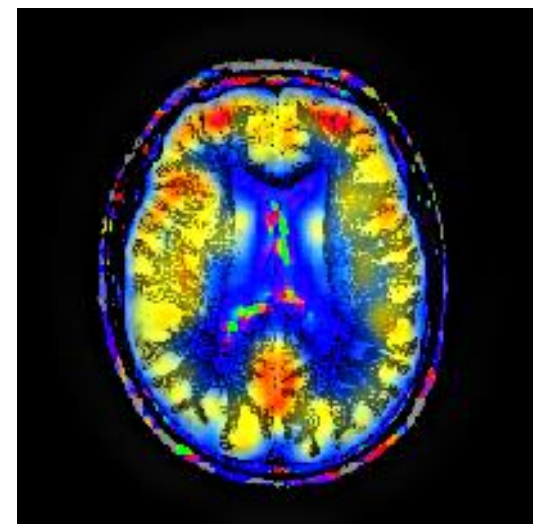

Figure 3. Alzhemier's disease MRI and PET images (A and B), Brovey method (C), Haar Wavelet (D), MSVD (E), and Proposed method (IHS-NSCT-PCA) (F).

Table. I:Performance indices for comparison of MRI-PET fused images with existing and proposed method for Alzheimer's disease

\begin{tabular}{|l|c|c|c|c|c|c|c|c|}
\hline Fusion Methods & \multicolumn{1}{|c|}{ EN } & \multicolumn{1}{|c|}{ SD } & \multicolumn{1}{|c|}{ AG } & \multicolumn{1}{c|}{ D } & \multicolumn{1}{c|}{ OP } & \multicolumn{1}{c|}{ AD } & SC & NCC \\
\hline Brovey & 3.736395 & 0.276632 & 0.0337 & 0.159923 & 0.126223 & 0.04602 & 1.172273 & 0.500189 \\
\hline HaarWavelet & 2.710903 & 0.269523 & 0.050225 & 0.162603 & 0.109377 & 0.080541 & 1.786031 & 0.326744 \\
\hline MSVD & 3.356959 & 0.343888 & 0.049912 & 0.150554 & 0.100642 & 0.027269 & 1.112873 & 0.484332 \\
\hline IHS-NSCT-PCA & $\mathbf{4 . 7 8 1 6 3 1}$ & $\mathbf{0 . 3 7 2 7}$ & $\mathbf{0 . 0 5 3 5 6 4}$ & $\mathbf{0 . 1 0 8 4 7 3}$ & $\mathbf{0 . 0 5 7 9 0 9}$ & $\mathbf{- 0 . 0 3 8 5 8}$ & $\mathbf{0 . 6 2 0 3 1 5}$ & $\mathbf{0 . 7 3 4 2 6 3}$ \\
\hline
\end{tabular}

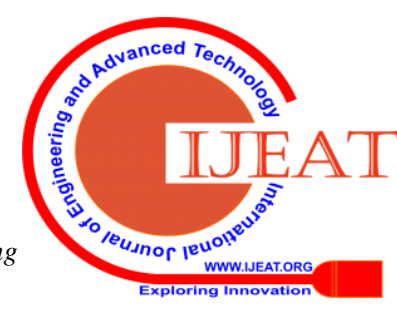


(A)

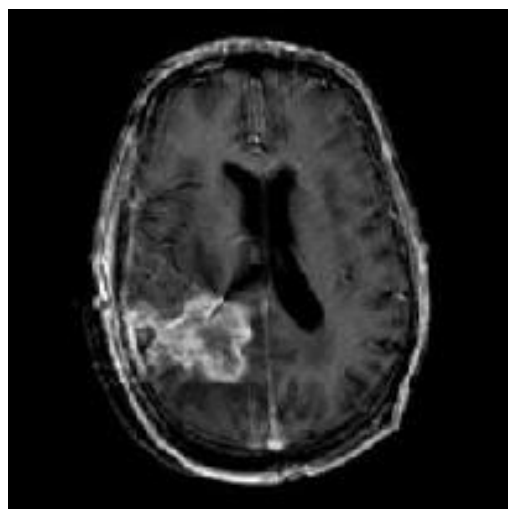

(C)

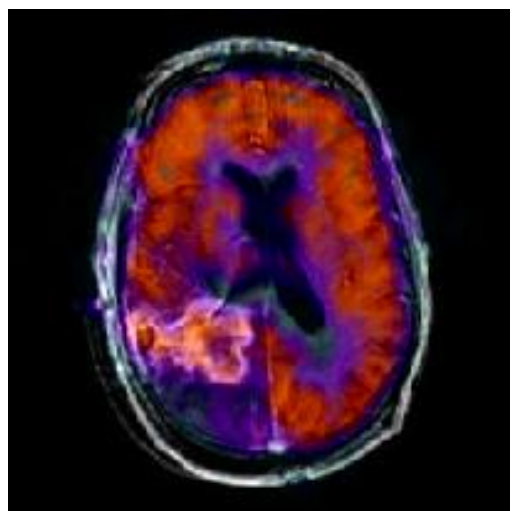

(E)

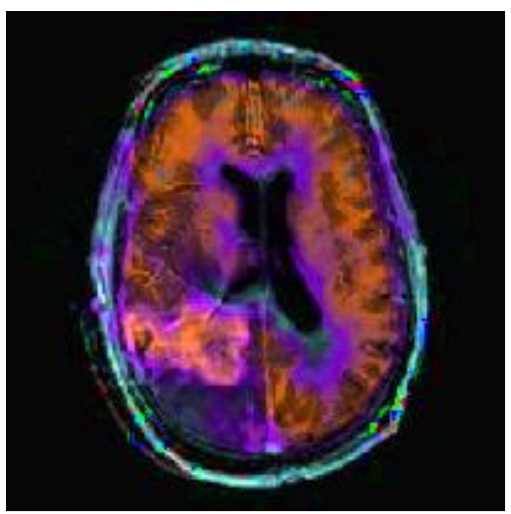

(B)

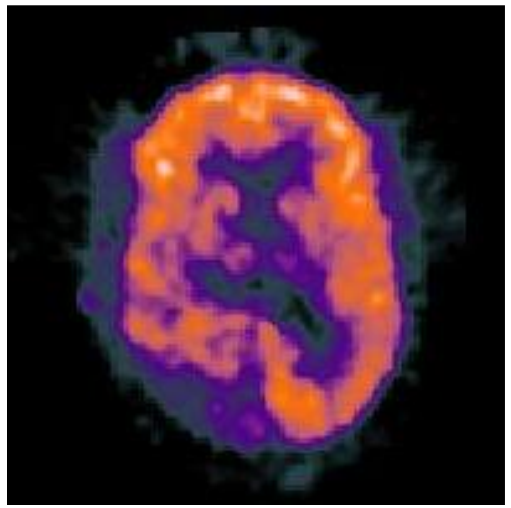

(D)

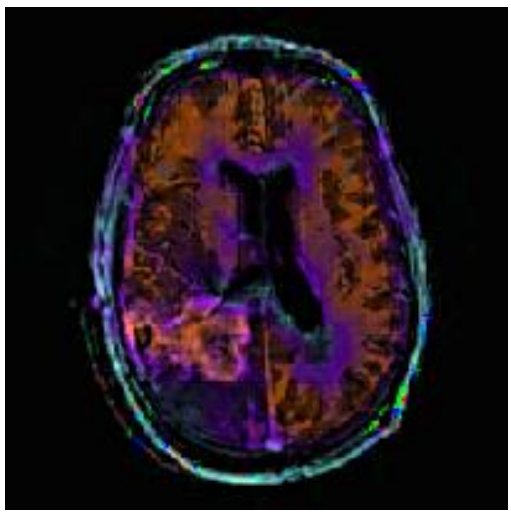

(F)

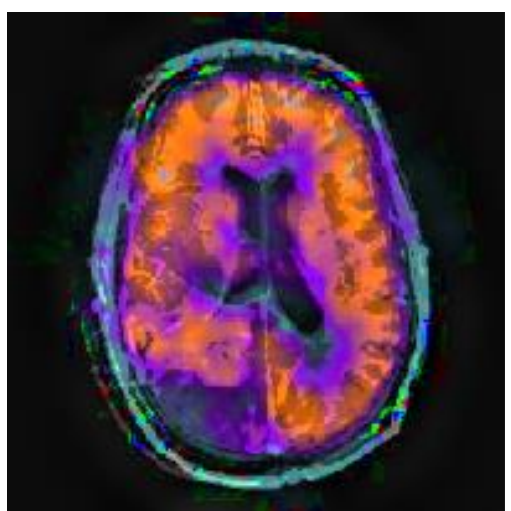

Figure 4. Brain MRI and PET images with tumor (A and B), Brovey method (C), Haar Wavelet (D), MSVD (E) and proposed method (IHS-NSCT-PCA) (F).

Table. II: Performance indices for comparison of MRI-PET fused images with existing and proposed method for Brain tumor

\begin{tabular}{|l|c|l|c|c|c|c|c|c|}
\hline Fusion Methods & \multicolumn{1}{|c|}{ EN } & \multicolumn{1}{c|}{ SD } & \multicolumn{1}{c|}{ AG } & \multicolumn{1}{c|}{ D } & \multicolumn{1}{c|}{ OP } & \multicolumn{1}{c|}{ AD } & SC & NCC \\
\hline Brovey & 4.669336 & 0.2135 & 0.023097 & 0.104476 & 0.081379 & 0.036942 & 1.305591 & 0.696016 \\
\hline Haar Wavelet & 4.815938 & 0.153872 & 0.026347 & 0.1244 & 0.098053 & 0.066016 & 2.109906 & 0.509861 \\
\hline MSVD & 5.352016 & 0.211795 & 0.025947 & 0.101536 & 0.07559 & 0.001026 & 1.007918 & 0.801159 \\
\hline IHS-NSCT-PCA & $\mathbf{6 . 6 2 0 9 9 2}$ & $\mathbf{0 . 2 4 4 9 2 9}$ & $\mathbf{0 . 0 2 7 0 2 7}$ & $\mathbf{0 . 0 8 4 6}$ & $\mathbf{0 . 0 5 7 5 7 3}$ & $\mathbf{- 0 . 0 5 4 8 4}$ & $\mathbf{0 . 6 9 8 4 1 4}$ & $\mathbf{1 . 0 2 5 1 9}$ \\
\hline
\end{tabular}

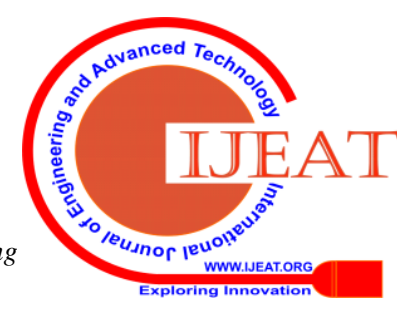




\section{CONCLUSION}

The ultimate determination of multimodal medical image fusion is to increase the imaging quality and clarity, obtain rich information content, and reduce the storage space. Most of the time, the emphasis is on the extraction of features at various scales, edges, corners and orientations within the images. Hence NSCT is integrated with IHS-PCA method to extract significant data from fused images. Fusion results can help the physicians for better diagnosis. The spatial resolution of PET image is low and has pseudo color. There is no color information content in MRI image and has good spatial resolution.In this work, we have integrated IHS transform to preserve spatial information, PCA to minimize redundancy and NSCT to display the spectral contents inmultiscale and multidirections to obtainthe fused image. Hence the proposed IHS-NSCT-PCA integrated technique outperforms the existing spatial and transforms domain methods.It also gives best results with regard to feature reduction for storage, reduced computational complexity, human visualization and objective evaluation.

\section{ACKNOWLEDGMENT}

It is our pleasure and kindness tohttp://www. med.harvard. edu/annlib/, "The Whole Brain Atlas Database" of Harvard medical school, for providing access to the database. Special thanks to Dr.Adithi Jain, Assistant Professor, Radio diagnosis department, Ramaiah Medical College, Bengaluru, for her valuable guidance and suggestions regarding various imaging modalities and their application in identifying pathologies.

\section{REFERENCES}

1. A. P. James and B. V Dasarathy, 'Medical Image Fusion : A survey of the state of the art', Inf. Fusion, vol. 19, pp. 4-19, 2014.

2. S. Goyal and R. Wahla, 'A Survey on Image Fusion', pp. 7574-7581, 2015.

3. K. Yeshwant and M. Nanded, 'The IHS Transformations Based Image Fusion', no. July 2011, 2014

4. C. He, Q. Liu, H. Li, and H. Wang, 'Multimodal medical image fusion based on IHS and PCA', in Procedia Engineering, 2010, vol. 7, pp. 280-285.

5. P. Santhi, G. Thirugnanam, P. Mangaiyarkarasi, I. Engg, and T. Nadu, 'Image Fusion Technique for Multi-Resolution Medical Images Using Directional Contourlet Transform', vol. 34, no. 9, pp. 1177-1182, 2016.

6. W. Dou and Y. Chen, 'AN IMPROVED IHS IMAGE FUSION METHOD', no. 3, pp. 1253-1256.

7. T. M. Tu, S. C. Su, H. C. Shyu, and P. S. Huang, 'A new look at IHS-like image fusion methods', Inf. Fusion, vol. 2, no. 3, pp. 177-186, 2001.

8. T. Tu, S. Su, H. Shyu, and P. S. Huang, 'Efficient intensity-hue-saturation-based image fusion with saturation compensation', 2001

9. J. Du, W. Li, K. Lu, and B. Xiao, 'Neurocomputing An overview of multi-modal medical image fusion', vol. 215, pp. 3-20, 2016.

10. M. N. Do and M. Vetterli, 'Contourlets', Stud. Comput. Math., vol. 10, pp. 83-105, 2003.

11. A. L. da Cunha, J. Zhou, and M. N. Do, 'The nonsubsampled contourlet transform: Theory, design, and applications', IEEE Trans. Image Process., vol. 15, no. 10, pp. 3089-3101, 2006.

12. S. Serikawa, H. Lu, Y. Li, L. Zhang, and S. Yang, 'Multimodal Medical Image Fusion in Non-Subsampled Contourlet Transform Domain', Circuits Syst., vol. 7, no. June, pp. 1598-1610, 2016.

13. M. Nazrudeen, M. Rajalakshmi, and S. Sureshkumar, 'Image fusion using nonsubsampled contourlet transform', Int. J. Eng. Res. Technol., vol. 3, no. 3, pp. 719-724, 2014.

14. N. Amini, E. Fatemizadeh, and H. Behnam, 'MRI-PET image fusion based on NSCT transform using local energy and local variance fusion rules', J. Med. Eng. Technol., vol. 38, no. 4, pp. 211-219, 2014.

15. P. S. Gomathi and B. Kalaavathi, 'Multimodal Medical Image Fusion in Non-Subsampled Contourlet Transform Domain', no. June, pp. 1598-1610, 2016.

16. .P. Ganasala and V. Kumar, 'CT and MR image fusion scheme in nonsubsampled contourlet transform domain', J. Digit. Imaging, vol. 27, no. 3, pp. 407-418, 2014.

17. G. Bhatnagar, Q. M. J. Wu, and Z. Liu, 'Directive contrast based multimodal medical image fusion in NSCT domain', IEEE Trans. Multimed., vol. 15, no. 5, pp. 1014-1024, 2013.

18. G. Bhatnagar, Q. M. J. Wu, and Z. Liu, 'A new contrast based multimodal medical image fusion framework', Neurocomputing, vol. 157, pp. 143-152, 2015.

19. A. M. Eskicioglu and P. S. Fisher, 'Image Quality Measures and Their Performance - Communications, IEEE Transactions on', vol. 43, no 12, pp. 2959-2965, 1995.

20. P. Jagalingam and A. V. Hegde, 'A Review of Quality Metrics for Fused Image', Aquat. Procedia, vol. 4, no. Icwrcoe, pp. 133-142, 2015.

21. 21.S. S. N. College, 'Research Article A Survey on Quantitative Metrics for Assessing the Quality of Fused Medical Images S . Kavitha and $2 \mathrm{~K}$. K . Thyagharajan Department of ECE , RMD Engineering College, Chennai-601 206 , Tamilnadu , India', vol. 12, no. 3, pp. 282-293, 2016.

22. V. P. S. Naidu, 'Image Fusion Technique using Multi-resolution Singular Value Decomposition', Def. Sci. J., vol. 61, no. 5, p. 479, 2011.

23. K. Padmavathi, M V Karki, Multimodal Medical Image Fusion using IHS-DTCWT-PCA integrated Approach for Extracting Tumo Features., Int.J.Adv. Res. Comput. Sci, 9(2), pp.35-39, (2018).

24. http://www.med.harvard.edu/aanlib/,Keith A Johnson, J Alex Becker

\section{AUTHORS PROFILE}

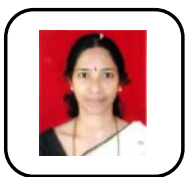

Padmavathi K. perused her B.E degree from Mangalore University in 2000 and M.Tech degree from VTU, Belagavi in 2007. She is currently working as an Associate Professorin the department of E\&C at NMAMIT, Nitte. She has an experience of 19 years in the field of teaching. Her area of interest includes, Signal \& Image Processing, Medical Image Processing, Pattern Recognition and Machine Learning.

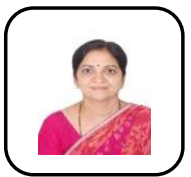

Dr. Maya V. Karkipersued her B.E Degree from Karnataka University, in 1986, M. Tech degree from Bangalore University in 1991 and Ph. D from VTU, Belagai in 2013. She is currently working as a Professor in the department of E\&C at Ramaiah Institute of Technology, Bengaluru. Her areas of interest include, Image \& Signal Processing, Video Analytics, machine Learning, Neura Networks and Multimedia Communication. She has many conference papers and journal papers to her credit. 